\title{
The significant prognostic value of circulating tumor cells in triple-negative breast cancer: a meta-analysis
}

\author{
Yan-jun Lu, ${ }^{1, *}$, Peng Wang ${ }^{2, *}$, Xiong Wang ${ }^{1}$, Jing Peng ${ }^{1}$, Yao-wu Zhu ${ }^{1}$ and Na Shen ${ }^{1}$ \\ ${ }^{1}$ Department of Laboratory Medicine, Tongji Hospital, Tongji Medical College, Huazhong University of Science and Technology, \\ Wuhan, China \\ ${ }^{2}$ Institute and Department of Infectious Disease, Tongji Hospital, Tongji Medical College, Huazhong University of Science \\ and Technology, Wuhan, China \\ * These authors have contributed equally to this work
}

Correspondence to: Na Shen, email: shenna@tjh.tjmu.edu.cn

Keywords: triple-negative breast cancer (TNBC); circulating tumor cells (CTCs); prognosis

Received: September 18, 2015 Accepted: February 29, 2016

Published: March 17, 2016

\section{ABSTRACT}

Background: The clinical validity of circulating tumor cells (CTCs) is still controversial in patients with triple-negative breast cancer (TNBC).

Methods: A comprehensive literature search was performed to identify relevant articles in the PubMed, Web of Science, MEDLINE, and Embase databases through September 2015. The outcomes of interest were disease progression and overall survival. The hazard ratio (HR) and $95 \%$ confidence interval (95\% CI) were considered the effect indicators and were pooled in meta-analyses under a fixed- or random-effect model according to heterogeneity.

Results: Ten of the eligible studies were included for a total of 642 enrolled TNBC patients. Overall analyses revealed that the presence of CTCs predicted aggressive disease progression $\left(H R=2.18,95 \% C I=1.59-2.99, P_{\text {heterogeneity }}=0.010, I^{2}=52.2 \%\right)$ and reduced overall survival $\left(\mathrm{HR}=2.02,95 \% \mathrm{CI}=1.59-2.57, P_{\text {heterogeneity }}=0.169, I^{2}=\right.$

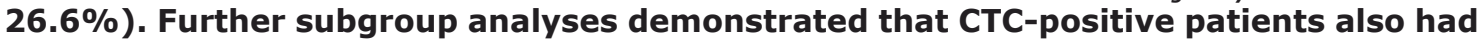
poor disease progression and overall survival in different subsets, including cancer stage.

Conclusion: Our meta-analysis provides strong evidence that detection of CTC in the peripheral blood is an independent prognosticator of poor survival outcomes for TNBC patients.

\section{INTRODUCTION}

Breast cancer is the most commonly diagnosed cancer and the leading cause of cancer-related mortality in women worldwide [1]. As a distinct subtype accounting for $10-20 \%$ of all breast cancers, triple-negative breast cancer (TNBC) lacks the expression of the estrogen receptor (ER), progesterone receptor (PR), and human epidermal growth factor receptor-2 (HER-2), resulting in an aggressive phenotype for which there is no targeted therapy [2]. Traditionally, these patients are treated with a combination of surgery, chemotherapy, and radiation. Finding an effective and non-invasive method for survival prediction is clinically important to the management of TNBC patients.

Circulating tumor cells (CTCs) are cells released from the primary tumor into the peripheral blood and are considered to be a main cause of tumor metastasis [3]. In recent years, accumulating evidence has suggested the prognostic relevance of CTCs in several malignancies, such as breast cancer [4], colorectal cancer [5] and melanoma [6]. However, the prognostic value of CTC status in TNBC remains unclear. Some studies revealed that CTC status could predict poorer survival outcomes in TNBC [7-11], while other studies failed to support this conclusion [12-14]. In addition, studies by Giordano et al. [15] and Hwang et al. [16] showed that CTC status had prognostic relevance for overall survival but not for disease progression in TNBC. Karhade and his colleagues [17] found conflicting results using various cut-off criteria. Interestingly, even in the same trial, CTCs detected at different time points indicated different prognoses for 
Table 1: Characteristics of studies included in our meta-analysis

\begin{tabular}{|c|c|c|c|c|c|c|c|c|c|c|c|}
\hline Study & Country & $\begin{array}{l}\text { Sample } \\
\text { size }\end{array}$ & Sampling time & $\begin{array}{l}\text { Median } \\
\text { follow-up }\end{array}$ & Stage & $\begin{array}{l}\text { Detection } \\
\text { method }\end{array}$ & $\begin{array}{l}\text { Detection } \\
\text { rate, } \%(\mathrm{n} / \mathrm{N})\end{array}$ & Cut-off criteria & Outcomes & HR extraction & $\begin{array}{l}\text { Multivariate } \\
\text { adjustment }\end{array}$ \\
\hline Ignatiadis (2007) & Greece & 77 & baseline & $53.5^{\mathrm{a}}$ & M0 & RT-PCR & $35(27 / 77)$ & - & DFS; OS & Data extrapolated & No \\
\hline Mego (2011) & USA & 72 & baseline & $17.7^{\mathrm{a}}$ & M1 & CellSearch & $57(41 / 72)$ & $1 \mathrm{CTC} / 7.5 \mathrm{ml}$ & OS & Reported in text & No \\
\hline Giordano (2012) & USA & 124 & baseline & $24.6^{\mathrm{a}}$ & M1 & CellSearch & $34(42 / 124)$ & $5 \mathrm{CTC} / 7.5 \mathrm{ml}$ & PFS; OS & Data extrapolated & No \\
\hline Hwang (2012) & Korea & 36 & baseline & $100.6^{\mathrm{a}}$ & Mo & RT-PCR & $25(9 / 36)$ & - & MFS; OS & Data extrapolated & No \\
\hline Munzone (2012) & Italy & 18 & baseline & $42^{a}$ & M1 & CellSearch & $22(4 / 18)$ & $5 \mathrm{CTC} / 7.5 \mathrm{ml}$ & OS & Reported in text & Yes \\
\hline \multirow[t]{3}{*}{ Jiang (2013) } & China & 39 & baseline & NR & M1 & CellSearch & $46(18 / 39)$ & $5 \mathrm{CTC} / 7.5 \mathrm{ml}$ & PFS; OS & Reported in text & Yes \\
\hline & & 25 & $\begin{array}{l}\text { mid-therapy } \\
\text { (1st follow-up) }\end{array}$ & NR & M1 & CellSearch & $16(4 / 25)$ & $5 \mathrm{CTC} / 7.5 \mathrm{ml}$ & PFS; OS & Reported in text & Yes \\
\hline & & 24 & $\begin{array}{l}\text { mid-therapy } \\
\text { (2nd follow-up) }\end{array}$ & NR & M1 & CellSearch & $17(4 / 24)$ & $5 \mathrm{CTC} / 7.5 \mathrm{ml}$ & PFS; OS & Reported in text & Yes \\
\hline Karhade (2014) & USA & 113 & baseline & 40 & M0 & CellSearch & $20(23 / 113)$ & $1 \mathrm{CTC} / 7.5 \mathrm{~mL}$ & PFS; OS & Reported in text & No \\
\hline Peeters (2014) & Belgium & 16 & baseline & $27.7^{\mathrm{a}}$ & M1 & CellSearch & $50(8 / 16)$ & $5 \mathrm{CTC} / 7.5 \mathrm{ml}$ & PFS; OS & Reported in text & No \\
\hline \multirow[t]{4}{*}{ Magbanua (2015) } & USA & 95 & baseline & 26 & M1 & CellSearch & $44(42 / 95)$ & $5 \mathrm{CTC} / 7.5 \mathrm{ml}$ & TTP; OS & Reported in text & Yes \\
\hline & & 89 & mid-therapy & 26 & M1 & CellSearch & $33(29 / 89)$ & $5 \mathrm{CTC} / 7.5 \mathrm{ml}$ & TTP; OS & Reported in text & Yes \\
\hline & & 91 & baseline & 26 & M1 & $\mathrm{IE} / \mathrm{FC}$ & $33(30 / 91)$ & $5 \mathrm{CTC} / 7.5 \mathrm{ml}$ & TTP; OS & Reported in text & Yes \\
\hline & & 82 & mid-therapy & 26 & M1 & $\mathrm{IE} / \mathrm{FC}$ & $34(28 / 82)$ & $5 \mathrm{CTC} / 7.5 \mathrm{ml}$ & TTP; OS & Reported in text & Yes \\
\hline \multirow[t]{3}{*}{ Paoletti (2015) } & USA & 52 & baseline & NR & M1 & CellSearch & $36(19 / 52)$ & $5 \mathrm{CTC} / 7.5 \mathrm{ml}$ & PFS & Reported in text & No \\
\hline & & 52 & $\begin{array}{l}\text { mid-therapy } \\
\text { (day } 15 \text { ) }\end{array}$ & NR & M1 & CellSearch & $27(14 / 52)$ & $5 \mathrm{CTC} / 7.5 \mathrm{ml}$ & PFS & Reported in text & No \\
\hline & & 49 & $\begin{array}{l}\text { mid-therapy } \\
\text { (day 29) }\end{array}$ & NR & M1 & CellSearch & $26(13 / 49)$ & $5 \mathrm{CTC} / 7.5 \mathrm{ml}$ & PFS & Reported in text & No \\
\hline
\end{tabular}

Abbreviations: NR, not reported; M0, non-metastasis; M1, metastasis; RT-PCR, reverse transcriptase polymerase chain reaction; IE/FC, immunomagnetic enrichment/flow cytometric; DFS, disease-free survival; PFS, progression-free survival; MFS, metastasis-free survival; TTP, time to progression.

a The median follow-up referred to all breast cancer participants.

survival for TNBC participants [18]. These discrepancies may result from the small sample sizes used in these studies as well as differences in the sampling times, cutoff criteria, and detection methods used.

Although a previous pooled analysis explored the prognostic relevance of CTC status in metastatic breast cancer, including TNBC [19], the effect of CTC status on non-metastatic TNBC still requires clarification. Hence, we conducted this comprehensive meta-analysis to provide a better insight into the prognostic value of CTC status for patients with TNBC. Specifically, we evaluated the potential effects of CTC status (positive vs. negative) on disease progression outcomes (disease-free survival (DFS), progression-free survival (PFS), metastasisfree survival (MFS), and time to progression (TTP)) and overall survival (OS). Furthermore, we performed subgroup analyses, including cancer stage, time points of blood collection, detection method, sample size, detection rate, and cut-off criteria, to assess the potential effect of CTC status in these different subsets.

\section{RESULTS}

\section{Study characteristics}

Figure 1 provides an overview of the process used to select the studies. A total of 414 records were initially identified by the comprehensive literature search, comprising 410 records from databases screening and 4 records from previous reviews. After reviewing the titles and abstracts, 156 duplicates were filtered out, and 233 records were subsequently excluded because they were conference abstracts, irrelevant to CTCs or subtypes of breast cancer, review articles, or experimental studies. This left 25 full-text articles for further assessment, of which 15 studies were excluded for not including survival data for TNBC patients [20-26], not providing sufficient information to extrapolate hazard ratios (HRs) and 95\% confidence intervals (95\% CIs) [27-31], or including patients who overlapped with those in other trials $[8,9$, 32]. Finally, 10 eligible studies accounting for 642 TNBC patients were included for our meta-analysis [7, 10-18].

Early stage (M0) and metastatic stage (M1) TNBC patients were enrolled in $3[7,16,17]$ and 7 studies [1015, 18], respectively. Methods used for CTC detection included RT-PCR (reverse transcriptase polymerase chain reaction), CellSearch assay and IE/FC (immunomagnetic enrichment/flow cytometric) approaches. The detection rates for these methods ranged from $16 \%$ to $57 \%$. Two main cut-off values, $5 \mathrm{CTC} / 7.5 \mathrm{ml}$ and $1 \mathrm{CTC} / 7.5 \mathrm{ml}$, were used for CTC-counting methods. The characteristics of the eligible studies are summarized in Table 1.

\section{Overall analyses}

The HRs for disease progression (DFS, PFS, MFS, and TTP) were available in 8 studies [7, 10, 11, 14-18] accounting for 552 TNBC patients. In 3 studies [11, 14, $18]$, more than one HR was extracted from each trial by 
Table 2: Univariate meta-regression analysis for exploring potential sources of heterogeneity

\begin{tabular}{llll}
\hline & \multicolumn{3}{l}{ Disease progression $^{\mathbf{a}}$} \\
\cline { 2 - 4 } & Coefficient & SE & $\boldsymbol{P}$ \\
\hline Publication year & -0.04 & 0.08 & 0.629 \\
Sample size & -1.17 & 0.51 & 0.040 \\
Sampling time & 0.42 & 0.33 & 0.226 \\
Cancer stage & -0.6 & 0.44 & 0.195 \\
Detection method & -0.31 & 0.38 & 0.432 \\
Detection rate & -0.06 & 0.36 & 0.872 \\
Cut-off criteria & 1.05 & 1.08 & 0.355 \\
HR extraction & -0.15 & 0.42 & 0.720 \\
Multivariate adjustment & -0.49 & 0.31 & 0.143 \\
\hline
\end{tabular}

Abbreviations: SE, standard error of the coefficient.

${ }^{a}$ Disease profession outcomes including disease-free survival (DFS), progression-free survival (PFS), metastasis-free survival (MFS) and time to progression (TTP). The dependent variable is the $\ln \mathrm{HR}$ for DFS/PFS/ MFS/TTP or OS of each study.

using multiple sampling time points or different detection methods. The overall analysis revealed that compared with CTC-negative TNBC patients, the CTC-positive patients had a higher risk of disease progression $(\mathrm{HR}=2.18,95 \%$ $\left.\mathrm{CI}=1.59-2.99, P_{\text {heterogeneity }}=0.010, I^{2}=52.2 \%\right)($ Figure 2A). The HRs for OS were available in 9 studies [7, 10, 1218], accounting for 590 TNBC patients. More than one HR was extracted in 2 studies $[14,18]$ for the same reasons as mentioned above. The pooled results showed that CTCpositive TNBC patients also had significantly poorer outcomes than CTC-negative patients $(\mathrm{HR}=2.02,95 \% \mathrm{CI}$ $=1.59-2.57, P_{\text {heterogeneity }}=0.169, I^{2}=26.6 \%$ ) (Figure $\left.2 \mathrm{~B}\right)$.

\section{Meta-regression and subgroup analyses}

A significant heterogeneity among studies was observed when pooling the data for disease progression $\left(P_{\text {heterogeneity }}=0.010, I^{2}=52.2 \%\right)$, so we carried out a univariate meta-regression analysis to explore the potential sources. Several covariates, including publication year, sample size, and cancer stage, were considered, and the results indicated that sample size was the main factor responsible for the heterogeneity ( $P=0.040$; Table 2$)$.

To further assess whether CTC status had prognostic value in different subsets, we also performed subgroup analyses (Table 3). We first evaluated the effect of CTC status on outcomes for different cancer stages and found that for both M0 and M1 stages, detection of CTCs predicted a poor prognosis for both disease progression (M0: $\mathrm{HR}=3.51,95 \% \mathrm{CI}=1.90-6.48, P_{\text {heterogeneity }}=0.741$, $I^{2}=0.0 \% ; \mathrm{M} 1: \mathrm{HR}=1.99,95 \% \mathrm{CI}=1.42-2.81, P$ $\left.=0.011, I^{2}=55.0 \%\right)$ and OS (M0: HR $=4.18,95 \%$ CI $=2.02-8.62, P_{\text {heterogeneity }}=0.282, I^{2}=21.0 \% ; \mathrm{M} 1: \mathrm{HR}=$ $1.84,95 \% \mathrm{CI}=1.43-2.38, P_{\text {heterogeneity }}=0.370, I^{2}=7.8 \%$ ). We then explored the effects of CTC status on outcomes for various sampling times. CTCs detected at baseline indicated an increased risk for both disease progression $\left(\mathrm{HR}=1.86,95 \% \mathrm{CI}=1.27-2.72, P_{\text {heterogeneity }}=0.041, I^{2}\right.$ $=50.4 \%)$ and poor $\mathrm{OS}(\mathrm{HR}=2.19,95 \% \mathrm{CI}=1.67-2.87$, $\left.P_{\text {heterogeneity }}=0.307, I^{2}=14.8 \%\right)$. However, CTCs detected at mid-therapy exhibited prognostic significance for disease progression $\left(\mathrm{HR}=2.66,95 \% \mathrm{CI}=1.87-3.78, P_{\text {heterogeneity }}\right.$ $\left.=0.149, I^{2}=38.6 \%\right)$ but not for OS $(\mathrm{HR}=1.49,95 \%$ $\left.\mathrm{CI}=0.88-2.54, P_{\text {heterogeneity }}=0.134, I^{2}=46.2 \%\right)$. We also assessed the effects of CTC status on outcomes for various detection methods. CTCs detected by CellSearch assay or RT-PCR indicated a worse prognosis for both disease progression (CellSearch: $\mathrm{HR}=2.37,95 \% \mathrm{CI}=1.64-3.41$, $P_{\text {heterogeneity }}=0.018, I^{2}=53.4 \%$; RT-PCR: HR $=3.22,95 \%$ $\left.\mathrm{CI}=1.42-7.28, P_{\text {heterogeneity }}=0.480, I^{2}=0.0 \%\right)$ and $\mathrm{OS}$ (CellSearch: $\mathrm{HR}=2.21,95 \% \mathrm{CI}=1.66-2.93, P_{\text {heterogeneity }}$ $=0.796, I^{2}=0.0 \%$; RT-PCR: HR $=7.23,95 \% \mathrm{CI}=2.62-$ $19.97, P_{\text {heterogeneity }}=0.625, I^{2}=0.0 \%$ ). However, we did not find any significant prognostic effect of CTCs detected using IE/FC (For disease progression: $\mathrm{HR}=1.21,95 \% \mathrm{CI}$ $=0.55-2.68, P_{\text {heterogeneity }}=0.113, I^{2}=60.3 \%$; For OS: HR $=$ $\left.1.09,95 \% \mathrm{CI}=0.66-1.83, P_{\text {heterogeneity }}=0.728, I^{2}=0.0 \%\right)$. In addition, the effects of CTC status on outcomes were evaluated separately for different sample sizes, detection rates, and cut-off criteria. The stratified results showed that compared to CTC-negative patients, CTC-positive patients had a higher risk for both disease progression and OS in these subgroups (Table 3).

\section{Sensitivity analyses and publication bias}

For disease progression, one-way sensitivity analyses indicated the stability of our pooled results (Supplementary Table 1). Although publication bias existed $\left(P_{\text {Begg }}=0.042, P_{\text {Egger }}=0.011\right)$, the trim-and-fill 
Table 3: Subgroup analyses of the potential effect of CTCs on survival outcomes in TNBC patients

\begin{tabular}{|l|l|l|l|l|l|l|l|l|}
\hline \multicolumn{7}{|l}{ Disease progression ${ }^{\mathbf{a}}$} & \multicolumn{3}{l|}{ Overall survival } \\
\hline & $\mathrm{n}$ & $\mathrm{HR}(95 \% \mathrm{CI})$ & $P_{\text {hetergeneity }}$ & $I^{2}(\%)$ & $\mathrm{n}$ & $\mathrm{HR}(95 \% \mathrm{CI})$ & $P_{\text {hetergeneity }}$ & $I^{2}(\%)$ \\
\hline Cancer stage & & & & & & & & \\
\hline M0 & 3 & $3.51(1.90-6.48)$ & 0.741 & 0.0 & 3 & $4.18(2.02-8.62)$ & 0.282 & 21.0 \\
\hline M1 & 12 & $1.99(1.42-2.81)$ & 0.011 & 55.0 & 11 & $1.84(1.43-2.38)$ & 0.370 & 7.8 \\
\hline Sampling time & & & & & & & & \\
\hline baseline & 9 & $1.86(1.27-2.72)$ & 0.041 & 50.4 & 10 & $2.19(1.67-2.87)$ & 0.307 & 14.8 \\
\hline mid-therapy & 6 & $2.66(1.87-3.78)$ & 0.149 & 38.6 & 4 & $1.49(0.88-2.54)$ & 0.134 & 46.2 \\
\hline Detection method & & & & & & & & \\
\hline CellSearch & 11 & $2.37(1.64-3.41)$ & 0.018 & 53.4 & 10 & $2.21(1.66-2.93)$ & 0.796 & 0.0 \\
\hline IE/FC & 2 & $1.21(0.55-2.68)$ & 0.113 & 60.3 & 2 & $1.09(0.66-1.83)$ & 0.728 & 0.0 \\
\hline RT-PCR & 2 & $3.22(1.42-7.28)$ & 0.480 & 0.0 & 2 & $7.23(2.62-19.97)$ & 0.625 & 0.0 \\
\hline Sample size & & & & & & & & \\
\hline$<30$ & 3 & $5.96(2.53-14.04)$ & 0.595 & 0.0 & 4 & $3.99(1.73-9.18)$ & 0.636 & 0.0 \\
\hline$\geq 30$ & 12 & $1.93(1.43-2.61)$ & 0.033 & 47.7 & 10 & $1.90(1.47-2.44)$ & 0.153 & 31.9 \\
\hline Detection rate (\%) & & & & & & & & \\
\hline$<35$ & 10 & $2.32(1.49-3.63)$ & 0.003 & 64.5 & 9 & $1.89(1.40-2.56)$ & 0.222 & 24.9 \\
\hline$\geq 35$ & 5 & $2.09(1.44-3.04)$ & 0.460 & 0.0 & 5 & $2.27(1.52-3.39)$ & 0.161 & 39.1 \\
\hline Cut-off criteria & & & & & & & & \\
\hline 1 CTC/7.5 ml & 12 & $1.99(1.42-2.81)$ & 0.011 & 55.0 & 10 & $1.91(1.43-2.54)$ & 0.307 & 14.8 \\
\hline 5 CTC/7.5 ml & 0 & - & - & - & 2 & $1.76(1.07-2.89)$ & 0.525 & 0.0 \\
\hline
\end{tabular}

Abbreviations: IE/FC, immunomagnetic enrichment/flow cytometric; RT-PCR, reverse transcriptase polymerase chain reaction.

a Disease profession outcomes including disease-free survival (DFS), progression-free survival (PFS), metastasis-free survival (MFS) and time to progression (TTP).

analysis revealed that after incorporating 5 additional studies, the funnel plot was symmetrical, and the adjusted pooled HR was still similar to that in the main metaanalysis (Figure 2C; $\mathrm{HR}=1.75,95 \% \mathrm{CI}=1.27-2.41$ ). In addition, one-way sensitivity analyses for OS also confirmed our robust results (Supplementary Table 1), and the trim-and-fill analysis indicated that despite publication bias $\left(P_{\text {Begg }}=0.012, P_{\text {Egger }}=0.027\right)$, the adjusted pooled HR continued to show a significant association between CTC status and OS (Figure 2D; HR $=1.75,95 \% \mathrm{CI}=$ 1.39-2.20).

\section{DISCUSSION}

In this current meta-analysis, we performed a comprehensive literature search and provided clear evidence that CTCs detected in the blood can predict aggressive disease progression and poor OS for TNBC patients. Compared with a previous pooled analysis [19], our work not only included more subjects to support the clinical validity of CTC status in metastatic TNBC but also provided clear evidence to confirm the prognostic value of CTC status in non-metastatic TNBC. Moreover, we thoroughly evaluated the effects of CTC status on various sampling times, sample sizes, detection rates, and cut-off criteria. To the best of our knowledge, this is the first metaanalysis to comprehensively assess the prognostic value of CTC status for the TNBC phenotype.

When pooling the disease progression data, we observed significant heterogeneity among studies. The meta-regression analysis identified sample size as the primary source of this heterogeneity. However, the heterogeneity decreased or even disappeared after dividing subjects into small $(n<30)$ and large sample groups $(\geq 30)$. To explore the clinical utility of CTC status in TNBC, we also carried out subgroup analyses using different stratified factors. Although M0 and M1 patients had very different prognoses, the presence of CTCs indicated worse survival outcomes for TNBC. CTCs detected at baseline or midtherapy indicated a poor disease progression outcome. However, CTCs detected at mid-therapy failed to significantly predict poor OS, which may have been due to the insufficient statistical power. RT-PCR and CellSearch assay are two widely used methods for CTC detection. Our meta-analysis showed that CTCs detected by these two methods had a significant prognostic effect. However, for IE/FC, a new method developed by Magbanua et al. [14], the pooled HRs were not pronounced. Considering that the IE/FC group only included one study [14], more 


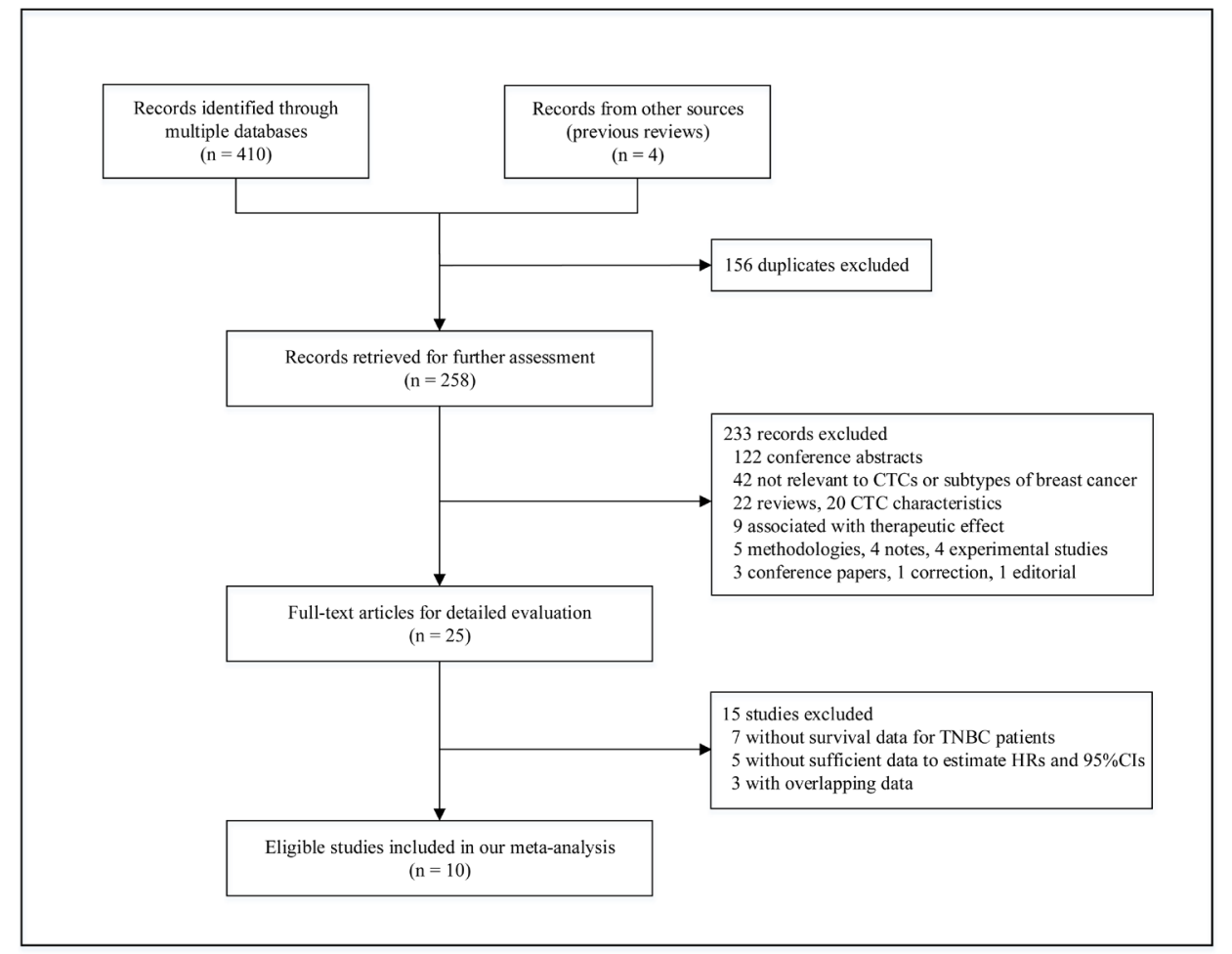

Figure 1: A flow chart of literature search.
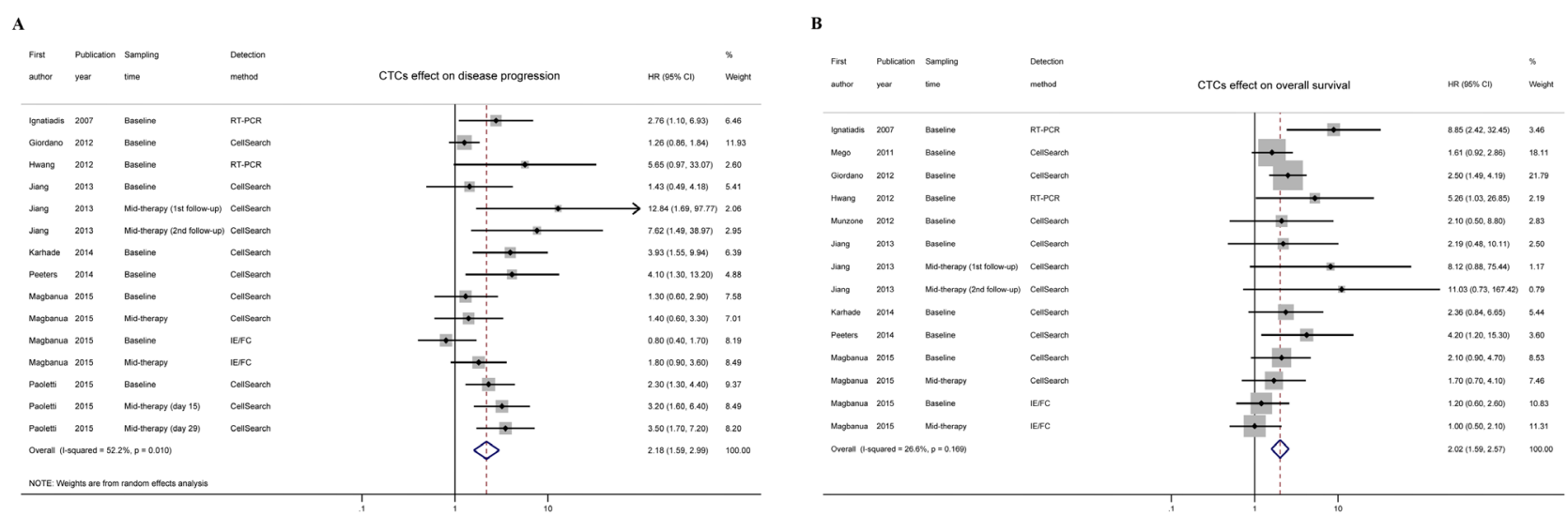

C

D
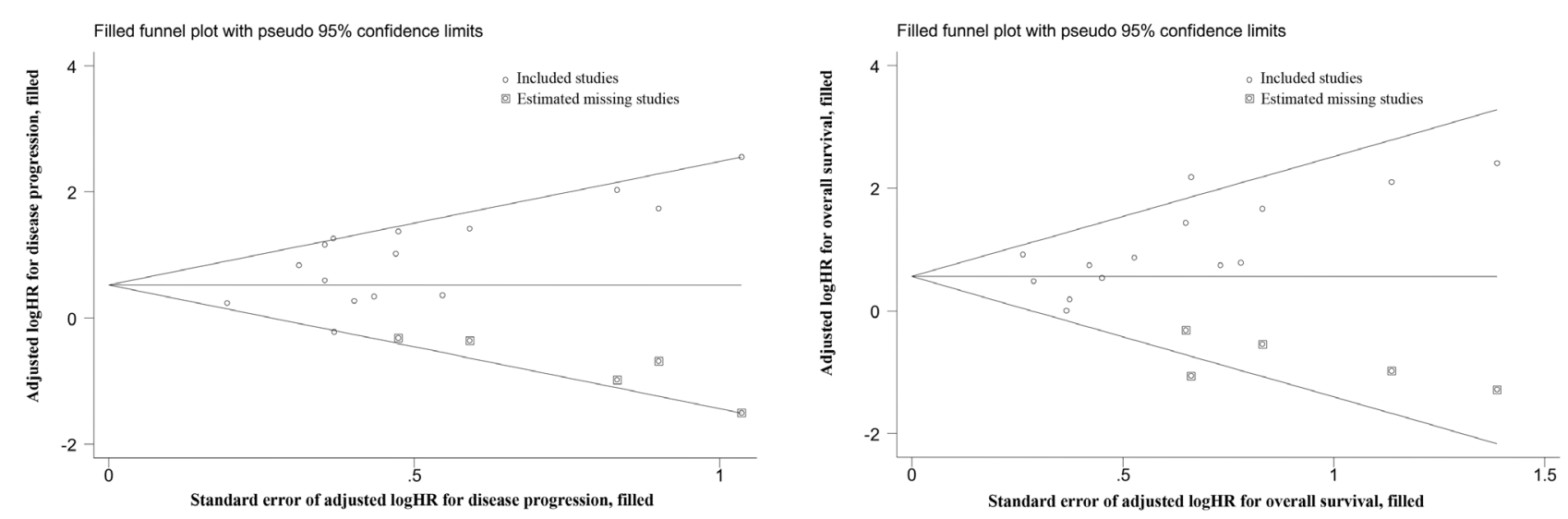

Figure 2: Overall analyses and imputed funnel plots. Forest plots of the HRs for disease progression A. and overall survival B. in TNBC patients. Imputed funnel plots by trim-and-fill analysis for disease progression $\mathbf{C}$. and overall survival $\mathbf{D}$. in TNBC patients. 
research is required to assess the effectiveness and stability of this method. The results from other subgroup analyses demonstrated that for different sample sizes, cut-off criteria, and detection rates, CTC-positive TNBC patients all had poor survival outcomes, suggesting that CTC status has a stable prognostic value.

Some limitations of this meta-analysis should be acknowledged. First, our meta-analysis was based on a comprehensive literature search, but we omitted data from several trials that did not provide sufficient information for HR extraction [27-31]. Although we were not able to extract HRs from these excluded trials, most of them suggested that CTC status had a significant prognostic value. Second, we did not acquire individual patient data from the included studies. These data, if available, might further improve the accuracy and stability of our pooled estimates. Third, obvious heterogeneity among studies was found for disease progression in our metaanalysis. Although the meta-regression analysis identified sample size as the only significant heterogeneous factor, variability in other influencing factors (e.g., study design and measurement of end points) might have contributed to the heterogeneity. Additional large-scale homogeneous studies are warranted to validate the clinical power of CTC status. Finally, the identified publication bias is of concern. We attempted to find all relevant articles, but unavoidably, some studies were likely omitted due to publication status or language restrictions. However, the trim-and-fill analysis showed that CTC status was still significantly associated with survival outcomes even when these "missing" studies were incorporated.

In summary, our meta-analysis provides strong support that detection of CTCs in the peripheral blood is an effective and promising predictor of a poor prognosis for TNBC patients. Regardless of whether CTCs are detected in an early stage or in metastatic patients, CTC status may serve as a useful tool to guide the clinical management of TNBC in the coming future. To improve the utility of CTC status in the management of TNBC, additional studies should be performed to further validate the prognostic power of CTCs detected during or after therapy and to develop a universally accepted standard method for CTC detection that has considerable sensitivity and stability.

\section{MATERIALS AND METHODS}

\section{Literature search}

A comprehensive electronic search was carried out using the PubMed, Web of Science, MEDLINE and Embase databases without any restriction (up to September 2015). The search items included various combinations of "breast", "cancer", "neoplasm", "carcinoma", "malign*”, "triple negative" and "circulating". The reference lists of the retrieved articles and reviews were also checked manually for potentially relevant studies. Only articles written in English published in peer-reviewed journals were included.

\section{Study selection}

Studies were considered eligible if they fulfilled all the following criteria: (1) retrospective or prospective cohort studies; (2) investigated the progression or survival of TNBC patients stratified by CTC status; (3) reported HRs and $95 \%$ CIs or provided sufficient information to extrapolate them. For studies with overlapping data, we only kept the study with the larger sample size. The process was performed independently by two authors, and any discrepancy was resolved by discussion or consultation with a third party if required. We documented the process via a flow chart as recommended by the PRISMA statement [33] (Supplementary Table 2). We did not assign a quality score to each study because no such score assessment has received a general consensus for use in non-randomized prognostic studies. Instead, we performed the widely recommended subgroup and sensitivity analyses to determine the potential effects of CTC status on the prognosis of TNBC patients.

\section{Data collection}

Two of the authors independently collected the following data from each eligible study: first author's name, publication year, country, number of subjects analyzed, cancer stage, median follow-up, timing of blood collection, detection method, detection rate, and cut-off value for CTC status. We also recorded the prognostic outcomes (DFS, PFS, MFS, TTP, OS, survival curves, $\mathrm{HR}$, and $95 \% \mathrm{CI}$, if available), regardless of whether they were tested by multivariate analysis. For one study [12], the reported HR indicated the CTC-negative rather than the CTC-positive arm. Thus we recalculated this HR by taking its reciprocal to maintain consistency with the other studies. When more than one blood sample per subject was collected at different time points, each sampling time point was documented and categorized as "baseline" or "mid- or post-therapy". When more than one method was applied to detect CTCs, all results were considered as independent data sets. Any discrepancy was resolved by discussion or consultation.

\section{Statistical methods}

The HR and 95\% CI were directly recorded from each included study or extrapolated as suggested by methods of Parmar [34] and Tierney [35]. We pooled these 
HRs using a fixed- or random-effect model according to heterogeneity [36]. Heterogeneity among studies was tested using Cochran's Q test and quantified by the $I^{2}$ index, which is considered significant if $P<0.10$ or $I^{2}$ $>50 \%$ by convention [37]. We then performed a metaregression analysis to investigate the potential causes of heterogeneity. Subgroup analyses were also conducted to determine the potentially prognostic effect of CTC status. In addition, publication bias was evaluated with Begg's and Egger's test, and its influence on the pooled HR was assessed by the "trim-and-fill" method [38-40]. To evaluate the stability of the pooled results, we carried out a one-way sensitivity analysis by recalculating the pooled HR after excluding each study in turn. All statistical tests were performed using Stata 12.1 software (College Station, TX, USA).

\section{CONFLICTS OF INTEREST}

The authors have no conflicts of interest to report.

\section{REFERENCES}

1. Ferlay J, Soerjomataram I, Dikshit R, Eser S, Mathers C, Rebelo M, Parkin DM, Forman D and Bray F. Cancer incidence and mortality worldwide: sources, methods and major patterns in GLOBOCAN 2012. International journal of cancer. 2015; 136:E359-386.

2. Diaz LK, Cryns VL, Symmans WF and Sneige N. Triple negative breast carcinoma and the basal phenotype: from expression profiling to clinical practice. Advances in anatomic pathology. 2007; 14:419-430.

3. Chaffer CL and Weinberg RA. A perspective on cancer cell metastasis. Science. 2011; 331:1559-1564.

4. Zhang L, Riethdorf S, Wu G, Wang T, Yang K, Peng G, Liu J and Pantel K. Meta-analysis of the prognostic value of circulating tumor cells in breast cancer. Clinical cancer research. 2012; 18:5701-5710.

5. Rahbari NN, Aigner M, Thorlund K, Mollberg N, Motschall E, Jensen K, Diener MK, Buchler MW, Koch M and Weitz J. Meta-analysis shows that detection of circulating tumor cells indicates poor prognosis in patients with colorectal cancer. Gastroenterology. 2010; 138:1714-1726.

6. Mocellin S, Hoon D, Ambrosi A, Nitti D and Rossi CR. The prognostic value of circulating tumor cells in patients with melanoma: a systematic review and meta-analysis. Clinical cancer research. 2006; 12:4605-4613.

7. Ignatiadis M, Xenidis N, Perraki M, Apostolaki S, Politaki E, Kafousi M, Stathopoulos EN, Stathopoulou A, Lianidou E, Chlouverakis G, Sotiriou C, Georgoulias V and Mavroudis D. Different prognostic value of cytokeratin-19 mRNA positive circulating tumor cells according to estrogen receptor and HER2 status in early-stage breast cancer. Journal of clinical oncology. 2007; 25:5194-5202.

8. Cristofanilli M, Broglio KR, Guarneri V, Jackson S,
Fritsche HA, Islam R, Dawood S, Reuben JM, Kau SW, Lara JM, Krishnamurthy S, Ueno NT, Hortobagyi GN, et al. Circulating tumor cells in metastatic breast cancer: biologic staging beyond tumor burden. Clinical breast cancer. 2007; 7:471-479.

9. Dawood S, Broglio K, Valero V, Reuben J, Handy B, Islam R, Jackson S, Hortobagyi GN, Fritsche H and Cristofanilli M. Circulating tumor cells in metastatic breast cancer: from prognostic stratification to modification of the staging system? Cancer. 2008; 113:2422-2430.

10. Peeters DJ, van Dam PJ, Van den Eynden GG, Rutten A, Wuyts H, Pouillon L, Peeters M, Pauwels P, Van Laere SJ, van Dam PA, Vermeulen PB and Dirix LY. Detection and prognostic significance of circulating tumour cells in patients with metastatic breast cancer according to immunohistochemical subtypes. British journal of cancer. 2014; 110:375-383.

11. Paoletti C, Li Y, Muniz MC, Kidwell KM, Aung K, Thomas DG, Brown ME, Abramson VG, Irvin WJ, Jr., Lin NU, Liu MC, Nanda R, Nangia JR, et al. Significance of Circulating Tumor Cells in Metastatic Triple-Negative Breast Cancer Patients within a Randomized, Phase II Trial: TBCRC 019. Clinical cancer research. 2015; 21:2771-2779.

12. Mego M, De Giorgi U, Dawood S, Wang X, Valero V, Andreopoulou E, Handy B, Ueno NT, Reuben JM and Cristofanilli M. Characterization of metastatic breast cancer patients with nondetectable circulating tumor cells. International journal of cancer. 2011; 129:417-423.

13. Munzone E, Botteri E, Sandri MT, Esposito A, Adamoli L, Zorzino L, Sciandivasci A, Cassatella MC, Rotmensz N, Aurilio G, Curigliano G, Goldhirsch A and Nole F. Prognostic value of circulating tumor cells according to immunohistochemically defined molecular subtypes in advanced breast cancer. Clinical breast cancer. 2012; 12:340-346.

14. Magbanua MJ, Carey LA, DeLuca A, Hwang J, Scott JH, Rimawi MF, Mayer EL, Marcom PK, Liu MC, Esteva FJ, Park JW and Rugo HS. Circulating tumor cell analysis in metastatic triple-negative breast cancers. Clinical cancer research. 2015; 21:1098-1105.

15. Giordano A, Giuliano M, De Laurentiis M, Arpino G, Jackson S, Handy BC, Ueno NT, Andreopoulou E, Alvarez RH, Valero V, De Placido S, Hortobagyi GN, Reuben JM, et al. Circulating tumor cells in immunohistochemical subtypes of metastatic breast cancer: lack of prediction in HER2-positive disease treated with targeted therapy. Annals of oncology. 2012; 23:1144-1150.

16. Hwang SB, Bae JW, Lee HY and Kim HY. Circulating Tumor Cells Detected by RT-PCR for CK-20 before Surgery Indicate Worse Prognostic Impact in TripleNegative and HER2 Subtype Breast Cancer. Journal of breast cancer. 2012; 15:34-42.

17. Karhade M, Hall C, Mishra P, Anderson A, Kuerer H, Bedrosian I, Krishnamurthy S and Lucci A. Circulating tumor cells in non-metastatic triple-negative breast cancer. 
Breast cancer research and treatment. 2014; 147:325-333.

18. Jiang ZF, Cristofanilli M, Shao ZM, Tong ZS, Song EW, Wang XJ, Liao N, Hu XC, Liu Y, Wang Y, Zeng L and Zhang M. Circulating tumor cells predict progressionfree and overall survival in Chinese patients with metastatic breast cancer, HER2-positive or triple-negative (CBCSG004): a multicenter, double-blind, prospective trial. Annals of oncology. 2013; 24:2766-2772.

19. Bidard FC, Peeters DJ, Fehm T, Nole F, Gisbert-Criado R, Mavroudis D, Grisanti S, Generali D, Garcia-Saenz JA, Stebbing J, Caldas C, Gazzaniga P, Manso L, et al. Clinical validity of circulating tumour cells in patients with metastatic breast cancer: a pooled analysis of individual patient data. The Lancet Oncology. 2014; 15:406-414.

20. Giordano A, Giuliano M, De Laurentiis M, Eleuteri A, Iorio F, Tagliaferri R, Hortobagyi GN, Pusztai L, De Placido S, Hess K, Cristofanilli M and Reuben JM. Artificial neural network analysis of circulating tumor cells in metastatic breast cancer patients. Breast cancer research and treatment. 2011; 129:451-458.

21. Pierga JY, Hajage D, Bachelot T, Delaloge S, Brain E, Campone M, Dieras V, Rolland E, Mignot L, Mathiot C and Bidard FC. High independent prognostic and predictive value of circulating tumor cells compared with serum tumor markers in a large prospective trial in first-line chemotherapy for metastatic breast cancer patients. Annals of oncology. 2012; 23:618-624.

22. Bidard FC, Belin L, Delaloge S, Lerebours F, Ngo C, Reyal F, Alran S, Giacchetti S, Marty M, Lebofsky R and Pierga JY. Time-Dependent Prognostic Impact of Circulating Tumor Cells Detection in Non-Metastatic Breast Cancer: 70-Month Analysis of the REMAGUS02 Study. International journal of breast cancer. 2013; 2013:130470.

23. Tryfonidis K, Boukovinas I, Xenidis N, Christophyllakis C, Papakotoulas P, Politaki E, Malamos N, Polyzos A, Kakolyris S, Georgoulias V and Mavroudis D. A multicenter phase I-II study of docetaxel plus epirubicin plus bevacizumab as first-line treatment in women with HER2-negative metastatic breast cancer. Breast. 2013; 22:1171-1177.

24. Zhao S, Yang H, Zhang M, Zhang D, Liu Y, Liu Y, Song Y, Zhang X, Li H, Ma W and Zhang Q. Circulating tumor cells (CTCs) detected by triple-marker EpCAM, CK19, and hMAM RT-PCR and their relation to clinical outcome in metastatic breast cancer patients. Cell biochemistry and biophysics. 2013; 65:263-273.

25. Wallwiener M, Riethdorf S, Hartkopf AD, Modugno C, Nees J, Madhavan D, Sprick MR, Schott S, Domschke C, Baccelli I, Schonfisch B, Burwinkel B, Marme F, et al. Serial enumeration of circulating tumor cells predicts treatment response and prognosis in metastatic breast cancer: a prospective study in 393 patients. BMC cancer. 2014; 14:512.

26. Helissey C, Berger F, Cottu P, Dieras V, Mignot L, Servois V, Bouleuc C, Asselain B, Pelissier S, Vaucher I, Pierga
JY and Bidard FC. Circulating tumor cell thresholds and survival scores in advanced metastatic breast cancer: the observational step of the CirCe01 phase III trial. Cancer letters. 2015; 360:213-218.

27. Muller V, Riethdorf S, Rack B, Janni W, Fasching PA, Solomayer E, Aktas B, Kasimir-Bauer S, Pantel K and Fehm T. Prognostic impact of circulating tumor cells assessed with the CellSearch System and AdnaTest Breast in metastatic breast cancer patients: the DETECT study. Breast cancer research. 2012; 14:R118.

28. Wallwiener M, Hartkopf AD, Baccelli I, Riethdorf S, Schott S, Pantel K, Marme F, Sohn C, Trumpp A, Rack B, Aktas B, Solomayer EF, Muller V, et al. The prognostic impact of circulating tumor cells in subtypes of metastatic breast cancer. Breast cancer research and treatment. 2013; 137:503-510.

29. Rack B, Schindlbeck C, Juckstock J, Andergassen U, Hepp P, Zwingers T, Friedl TW, Lorenz R, Tesch H, Fasching PA, Fehm T, Schneeweiss A, Lichtenegger W, et al. Circulating tumor cells predict survival in early averageto-high risk breast cancer patients. Journal of the National Cancer Institute. 2014; 106.

30. Madic J, Kiialainen A, Bidard FC, Birzele F, Ramey G, Leroy Q, Rio Frio T, Vaucher I, Raynal V, Bernard V, Lermine A, Clausen I, Giroud N, et al. Circulating tumor DNA and circulating tumor cells in metastatic triple negative breast cancer patients. International journal of cancer. 2015; 136:2158-2165.

31. Ueo H, Sugimachi K, Gorges TM, Bartkowiak K, Yokobori T, Muller V, Shinden Y, Ueda M, Ueo H, Mori M, Kuwano H, Maehara Y, Ohno S, et al. Circulating tumour cellderived plastin3 is a novel marker for predicting long-term prognosis in patients with breast cancer. British journal of cancer. 2015; 112:1519-1526.

32. Hall C, Karhade M, Laubacher B, Anderson A, Kuerer H, DeSynder S and Lucci A. Circulating Tumor Cells After Neoadjuvant Chemotherapy in Stage I-III Triple-Negative Breast Cancer. Annals of surgical oncology. 2015; 22 Suppl 3:552-558.

33. Moher D, Liberati A, Tetzlaff J and Altman DG. Preferred reporting items for systematic reviews and meta-analyses: the PRISMA Statement. Open medicine. 2009; 3:e123-130.

34. Parmar MK, Torri V and Stewart L. Extracting summary statistics to perform meta-analyses of the published literature for survival endpoints. Statistics in medicine. 1998; 17:2815-2834.

35. Tierney JF, Stewart LA, Ghersi D, Burdett S and Sydes MR. Practical methods for incorporating summary time-toevent data into meta-analysis. Trials. 2007; 8:16.

36. Harris RJ, Bradburn MJ, Deeks JJ, Harbord RM, Altman DG and Sterne JAC. metan: fixed- and random-effects meta-analysis. Stata Journal. 2008; 8:3-28.

37. Higgins JP and Thompson SG. Quantifying heterogeneity in a meta-analysis. Statistics in medicine. 2002; 21:1539-1558. 
38. Begg CB and Mazumdar M. Operating characteristics of a rank correlation test for publication bias. Biometrics. 1994; 50:1088-1101.

39. Egger M, Davey Smith G, Schneider M and Minder C. Bias in meta-analysis detected by a simple, graphical test. BMJ. 1997; 315:629-634.

40. Duval S and Tweedie R. Trim and fill: A simple funnelplot-based method of testing and adjusting for publication bias in meta-analysis. Biometrics. 2000; 56:455-463. 